Article

\title{
The Disk-Driven Jet of Cygnus A
}

\author{
Bia Boccardi *, Thomas P. Krichbaum and Uwe Bach
}

Max-Planck-Institut für Radioastronomie, 53121 Bonn, Germany; tkrichbaum@mpifr-bonn.mpg.de (T.P.K.); ubach@mpifr-bonn.mpg.de (U.B.)

* Correspondence: bboccardi@mpifr-bonn.mpg.de

Academic Editors: Jose L. Gomez, Alan P. Marscher and Svetlana G. Jorstad

Received: 5 September 2016; Accepted: 18 April 2017; Published: 28 April 2017

\begin{abstract}
Recently published results from VLBI observations at 3 and 7 millimeters of the radio galaxy Cygnus $\mathrm{A}$ are reviewed in this article, and discussed within the model of a prominently stratified jet outflow. At the source redshift $(z=0.056), \mathrm{mm}$-VLBI allows a spatial resolution down to 200 Schwarzschild radii to be achieved, providing an extremely detailed view of the two-sided jet base. Through a study of the kinematic properties of the flow and of its transverse structure, it is shown that the radio emission is produced by an accelerating, mildly relativistic, parabolically expanding disk-wind. The observed transverse stratification, both of the flux density and of the bulk speed, supports the presence of an invisible faster spine close to the jet axis, powered either by the inner regions of the accretion disk or by the spinning black hole.
\end{abstract}

Keywords: jets; high angular resolution; Cygnus A

\section{Introduction}

Since the early days of radio astronomy, the radio galaxy Cygnus A has been one of the favorite targets for studies aimed at understanding the physics of relativistic jets in AGN. Its proximity $\left(z=0.056, D_{\mathrm{L}}=249 \mathrm{Mpc}\right)$ and the jet orientation close to the plane of the sky $\left(\theta \sim 75^{\circ}\right)$ give us the unique opportunity to investigate a powerful Fanaroff Riley II object (FR II) at high spatial resolution and almost free from geometrical effects.

In addition to studies of the large scale structure and of the bright radio lobes characterizing it, Cygnus A is particularly suited for testing theoretical models describing the launch, acceleration and collimation of the outflow. Indeed, the scales where such mechanisms are expected to take place can be probed in this source through Very Long Baseline Interferometry (VLBI) observations at millimeter wavelengths. From an observational stand point, this is quite a rare possibility. According to theoretical arguments (e.g., [1,2]) and to predictions from GRMHD (General Relativistic Magneto-HydroDynamical) numerical simulations (e.g., [3,4]), the region where the initially magnetically dominated jet is accelerated, and its energy is converted into bulk kinetic energy, extends out to $10^{3}-10^{4}$ Schwarzschild radii $\left(R_{\mathrm{S}}\right)$ from the central engine. Even when exploiting interferometric techniques at the highest frequencies, the aforementioned region is usually unreachable by observers, as it lies upstream of the VLBI core in most of the classical blazars (e.g., [5]). The fact that most of the jets are already in equipartition, or even matter-dominated in the "blazar-zone", is supported by ample observational evidence, e.g., involving brightness temperature measurements [6] or modeling of the spectral energy distributions (SEDs) (see [7] for a discussion). Nearby radio galaxies, instead, have proven to be the optimal targets for investigating the jet launching region. For reasons which are not fully clarified [8] but most likely involve the reduced impact of projection effects, in radio galaxies the VLBI core appears to be located much closer to the central black hole than in blazars. For example, phase referencing observations of M 87 [9] have determined an offset of only 
14-23 $R_{\mathrm{S}}$ between the jet apex and the $43 \mathrm{GHz}$ core, while an upper limit of $100 R_{\mathrm{S}}$ could be inferred for NGC 1052 at $86 \mathrm{GHz}$ [10].

Observing jets so close to their vertex can also allow us to directly discriminate between different launching models, particularly if the jet base can be resolved transversely. Indeed, by measuring the transverse width in the vicinity of the jet apex, one can constrain the radial distance at which the flow is initially anchored. If it is larger than the inner radius of the accretion disk, defined by the location of the innermost stable circular orbit (ISCO), this indicates that the disk is, at least partially, driving the flow, as described in the work of Blandford and Payne [11]. A narrower width is expected, instead, if the jet is powered by the rotational energy of the black hole through the Blandford and Znajeck mechanism [12]. Again in M 87, VLBI observations at $1 \mathrm{~mm}$ with the Event Horizon Telescope [13] have revealed a narrow transverse size of $5.5 R_{\mathrm{S}}$, implying that the jet base is anchored close to the ISCO. In fact, the ISCO radius varies between 1 and $9 R_{\mathrm{S}}$ depending on the black hole spin parameter and on the orbit of the disk/black hole system.

In the following, we summarize results from a detailed mm-VLBI study of Cygnus A, first presented in Boccardi et al. 2016a, 2016b [14,15], and we interpret them in a model of a jet outflow with significant transverse stratification. The data set is comprised of four Global VLBI observations at $43 \mathrm{GHz}$ performed between 2007 and 2009 and three GMVA (Global Millimeter VLBI Array) observations at $86 \mathrm{GHz}$ from the period 2009-2010. Thanks to the much reduced synchrotron opacity at high radio frequencies and to an observing beam as small as $200 R_{\mathrm{S}}\left(\right.$ for $M_{\mathrm{BH}}=2.5 \times 10^{9} M_{\odot}$ [16]), we could resolve transversely the base of the two-sided flow and obtain a comprehensive picture of the jet parameters in the acceleration and collimation region. For a more exhaustive description of the data analysis and scientific discussion, the reader is referred to the original articles [14,15].

\section{Results}

\subsection{Kinematic Structure}

In Figure 1, a simplified model of Cygnus A at $43 \mathrm{GHz}$ is shown for each of the four epochs (October 2007, October 2008, March 2009, November 2009). This was obtained in DIFMAP by fitting circular Gaussian components to the self-calibrated visibilities through the subroutine MODELFIT. The source is well described by nine or ten features, and presents a pronounced emission gap west of $\mathrm{C} 1$.

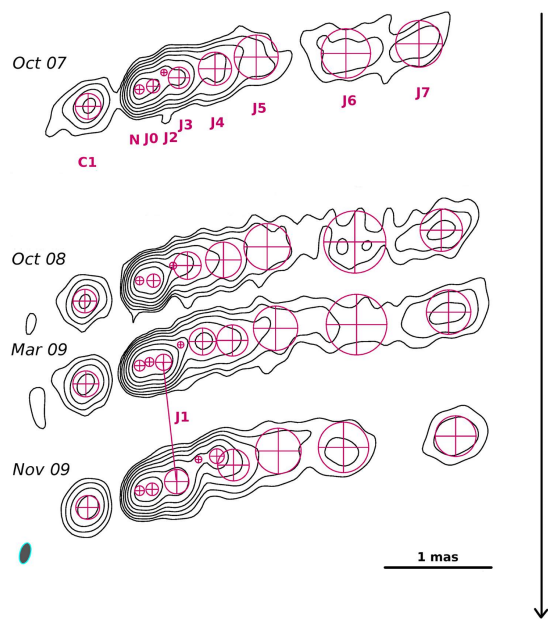

Figure 1. Modelfit maps of Cygnus A at $43 \mathrm{GHz}$. The images were tapered and convolved with a common beam with FWHM of $0.2 \times 0.1$ mas and position angle pa $=-15^{\circ}$. Contours represent isophotes at $1.8,3.6,7.2,14.4,28.8,57.6$, and $115.2 \mathrm{mJy} / \mathrm{beam}$. The reference point for the alignment is component N, assumed to be stationary [14]. Credit: Boccardi et al., A\&A, 585, A33 (2016), reproduced with permission (C)ESO. 
In order to measure the proper motions of the main emission features, these were cross-identified between different epochs based on a few main parameters (flux density, size, morphology), and each position was measured relative to component $\mathrm{N}$, assumed to be stationary. This analysis shows that while the motion of the features in the outer jet (J4, J5, J6, J7) is slower and uniform, and a linear fit is sufficient for determining the speeds, the inner components (J2 and J3) move faster and appear to accelerate, the motion being best described by second order polynomials. The two regions also show a different behavior when looking at the flux density variations of the components during the monitoring period. In the outer jet, the flux densities are constant, while closer to the core they sharply decrease as the components travel downstream. The latter also applies to the newly ejected feature J1. These results, as well as the jet limb-brightening observed in this source [14,17], can be well interpreted by assuming the presence of a transverse gradient of the bulk Lorentz factor of a spine-sheath kind. The decrease of the flux densities of the inner, accelerating components is likely caused by the decrease of their Doppler factor as the Lorentz factor increases. Unlike in blazars, this naturally occurs in jets seen at large viewing angles, not only in the counter-jet but also in the approaching side. If the speed is sufficiently high, the flow can be completely invisible, with a Doppler factor much smaller than 1. In this scenario, the lower speeds observed at larger distances from the core do not imply an intrinsic deceleration of the flow, but rather indicate that only the slower filaments are visible in that region. The de-boosting of a fast central spine has often been proposed as the possible cause of limb-brightening in jets. The analysis presented for Cygnus A provides direct evidence for it, since the flow is actually observed to get fainter and fainter as its speed increases.

In Figure 2, the intrinsic speeds $\beta$ and the Lorentz factors $\Gamma$ are reported as a function of distance $z$ from component $\mathrm{N}$. Two different colors are used for the fast and the slow sections of the flow, and the stationary features $\mathrm{C} 1$, J0 and J6 are also shown in green. A systematic increase of speed is observed along the jet, not only in the fast region but also in the slow one, although the individual components in the outer jet do not show clear signs of non-linear motion during the monitoring. The two sections accelerate with different gradients, at a faster rate closer to the jet axis than at the edge.
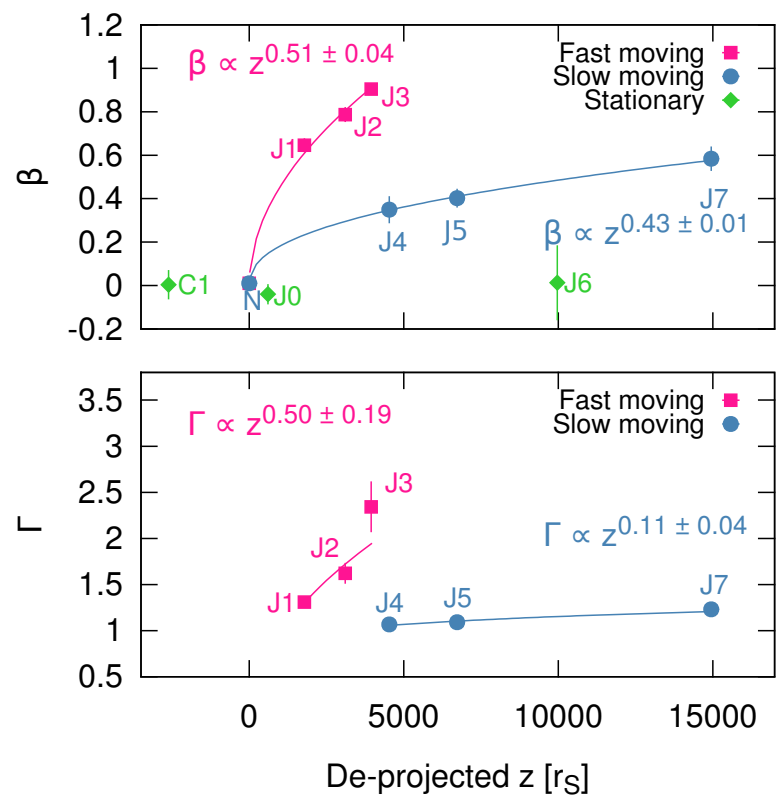

Figure 2. Top: intrinsic speed as a function of de-projected core separation. A fast and a slow component with different acceleration gradients can be identified; Bottom: corresponding Lorentz factor versus de-projected core separation [14]. Credit: Boccardi et al., A\&A, 585, A33 (2016), reproduced with permission (C)ESO. 
Overall, the acceleration region extends on a scale of $10^{3}-10^{4} R_{\mathrm{S}}$, which is in very good agreement with the expectations for a magnetically-driven jet (Section 1). The bulk Lorentz factor spans a mildly relativistic interval $(1<\Gamma<2.5)$, while an even faster but invisible flow may exist at the jet axis.

\subsection{Jet Shape}

By considering the transverse width of all the MODELFIT components in Figure 1, the jet shape was analyzed. As shown in Figure 3, the expansion can be described by a single power law of the form $r \propto z^{0.55 \pm 0.07}$ up to $\sim 10^{4} R_{S}$; i.e., the jet is almost perfectly parabolic. A gradual collimation of the flow, most likely provided by confinement from a poloidal magnetic field and/or from the external medium (see e.g., [18]), is found to be a necessary ingredient for a magnetically-driven jet to be efficiently accelerated. Almost the same expansion law was determined for M 87 on similar scales [19]. However, while in M 87 the jet becomes conical at larger distances, Cygnus A appears to have a cylindrical shape at low VLBI frequencies (e.g., [20]). Such a difference in the evolution of the two sources, FR I and FR II, respectively, may play an important role in the origin of the FR I/FR II dichotomy on sub-kiloparsec and kiloparsec scales. In fact, while magnetic acceleration is inefficient in a conical flow, it may still proceed in a flow attaining, asymptotically, a cylindrical shape. A fast, cylindrical jet would also be less affected by instabilities, and may propagate almost undisturbed until its final dissipation. According to [2,4], the steepness of the pressure gradient of the external medium crucially determines the asymptotic shape of the flow, and may therefore be a fundamental parameter for the development of the FR I/FR II dichotomy.

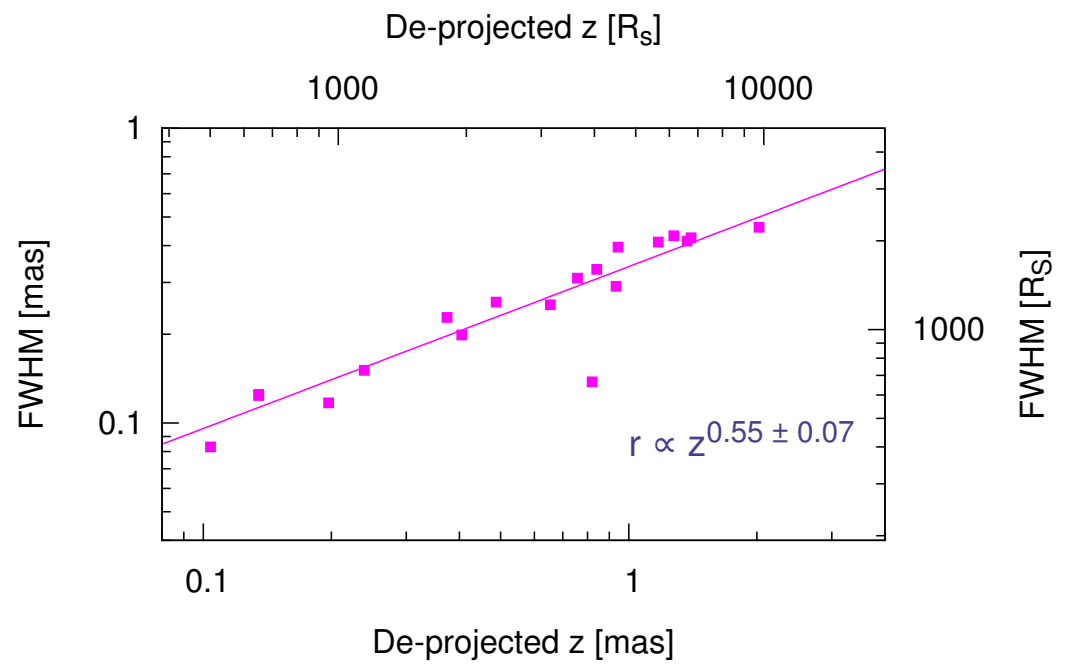

Figure 3. Power-law fit of the jet width (FWHM $=2 *$ radius $r$ ) versus de-projected distance from the core, as inferred from MODELFIT [14]. Credit: Boccardi et al., A\&A, 585, A33 (2016), reproduced with permission (C)ESO.

\subsection{Size of the Jet Base}

In order to zoom further into the jet launching regions of Cygnus A, three VLBI images with high dynamic range $(\sim 1200-2000)$ were produced at the frequency of $86 \mathrm{GHz}$. An angular resolution down to $\sim 45 \mu$ as, corresponding to a linear size of only $\sim 200$ Schwarzschild radii, could be achieved. In this article, Figure 4, we present the image obtained after stacking the three maps, a procedure which allows the best recovery of the jet structure. The morphological complexity of the source is apparent at this frequency. The emission is rich with sub-structures, and the limb-brightening seen at lower frequency can also be noticed, especially in the outer regions of the approaching jet. A prominent counter-jet is detected, while no clear emission gap is visible east of the core.

With the aim of determining the transverse width profile at the base of the two-sided flow, a pixel based analysis of the stacked map, which was convolved with a circular beam of 0.1 mas, was carried 
out in AIPS. With the task SLICE, the image was sliced along the jet axis; then, a Gaussian fit was performed with SLFIT for describing the transverse intensity distribution at each pixel ( $1 p x=0.02 \mathrm{mas})$. Results concerning the central 1.5 mas of the source are shown in Figure 5. The green dots indicate the measured transverse width $d_{\text {meas }}$ as a function of distance from the core $z$, while the black dotted line represents the profile of the formal resolution limit $d_{\text {lim, }}$ calculated based on the signal to noise ratio at each slice as [21]:

$$
d_{\lim }=\frac{4}{\pi} \sqrt{\pi \ln (2) b \ln \left(\frac{\left(S_{\mathrm{p}} / \sigma\right)}{\left(S_{\mathrm{p}} / \sigma\right)-1}\right)} .
$$

In this expression, $\sigma$ is the rms noise, $S_{\mathrm{p}}$ is the peak flux density at each pixel, and $b$ is the equivalent circular beam for the case of a uniform weighting. The comparison between the profiles in Figure 5 indicates that the measured width is much larger than the resolution limit, except for a very faint region at $z \sim-0.5$ mas.

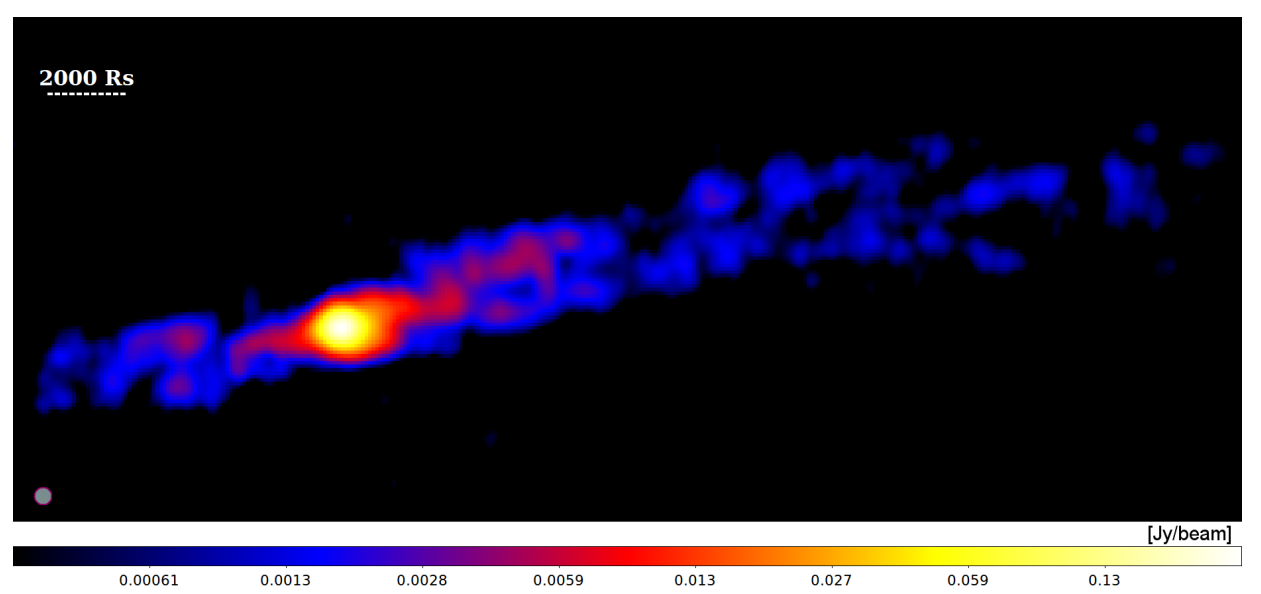

Figure 4. Stacked map of Cygnus A at $86 \mathrm{GHz}$. Each individual epoch was restored with a circular beam of 0.1 mas. The alignment was based on the position of the peak of intensity. The dashed line indicates a projected length of $\sim 2000$ Schwarzschild radii. The image is adapted from [15], Figure 2.

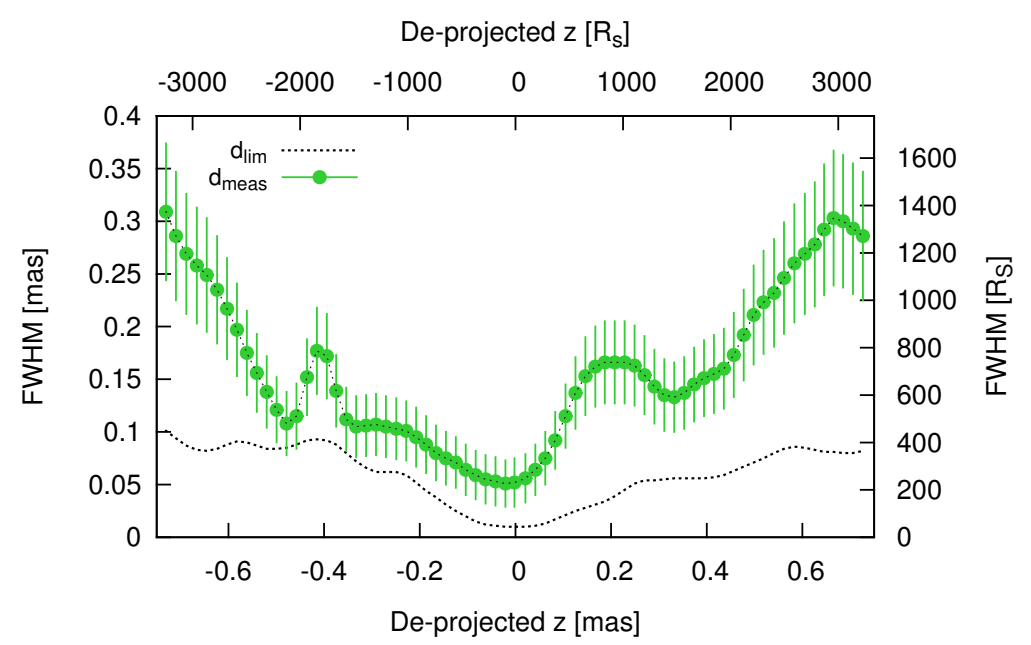

Figure 5. Transverse width profile at $86 \mathrm{GHz}$ for the stacked image. Error bars for the width measurements (green points with label $d_{\text {meas }}$ ) are assumed equal to $1 / 5$ of each convolved FWHM. The dashed black line with label $d_{\text {lim }}$ indicates the profile of the resolution limit [14]. Credit: Boccardi et al., A\&A, 588, L9 (2016), reproduced with permission (C)ESO. 
The base of the flow is therefore transversely resolved, which allows us to draw important conclusions from this analysis. The detection of a bright counter-jet provides us with the unique opportunity of inferring-assumptions free-a lower limit for the size of the jet base, even though the location of the central engine is not known exactly. Simply put, the central engine has to be "somewhere" between the jet and the counter-jet, and the transverse width of the flow at its origin cannot be smaller than the minimum size we measure. In units of Schwarzschild radii, the minimum is $\sim(227 \pm 98) R_{S}$, and it occurs in the surroundings of $z \sim 0$. This width is significantly larger than the radius of the innermost stable circular orbit, indicating that at least part of the flow is anchored at large disk radii in the accretion disk.

\section{Discussion}

Our VLBI study of Cygnus A at millimeter wavelengths provides a coherent description of the mechanisms taking place in the nuclear regions of this jet. As inferred from a detailed analysis at $86 \mathrm{GHz}$, the base of the two-sided flow is at least $\sim 230 R_{\mathrm{S}}$ wide, indicating that, unlike in M 87 [13], the outer regions of the accretion disk contribute in launching the jet. The radio emission is then produced by a disk wind which, based on the results obtained at $43 \mathrm{GHz}$, is of parabolic shape and accelerates on scales of $10^{3}-10^{4} R_{\mathrm{S}}$. The bulk Lorentz factor spans a mildly relativistic regime $(1<\Gamma<2.5)$, and shows a gradient also in the transverse direction. The complex kinematic properties of the source, as well as the jet limb-brightening, indicate the presence of a pronounced transverse stratification of a spine-sheath kind. Indeed, a fast central spine can be completely invisible, due to Doppler de-boosting, if seen at a sufficiently large viewing angle. This putative component of unknown speed (Figure 6) could be powered either by the inner regions of the accretion disk or by the spinning black hole itself. At the same time, the $86 \mathrm{GHz}$ jet may represent only a filament of an even broader disk wind extending to much larger radii, but resolved out at high resolution. Such a diffuse component is likely the main observable at low frequencies: at $5 \mathrm{GHz}$ [20], the jet of Cygnus A roughly appears as a cylinder and has a diameter of $\sim 9000 R_{S}$. After removing the broadening effect from scattering in the interstellar medium, this size may still be quite larger than measured at $86 \mathrm{GHz}$.

If such kind of stratification is a common feature of relativistic jets, Doppler boosting effects will naturally select the dominant component based on the jet orientation with respect to the observer. A frequency and/or resolution dependence may also be expected. Studies of large sample of blazars (e.g., [22]), i.e., of spine-dominated objects, show that the contribution of a spinning black hole is necessary for explaining the observed jet powers. In radio galaxies, instead, the mildly relativistic disk wind may be more easily observed, as in the case of Cygnus A.

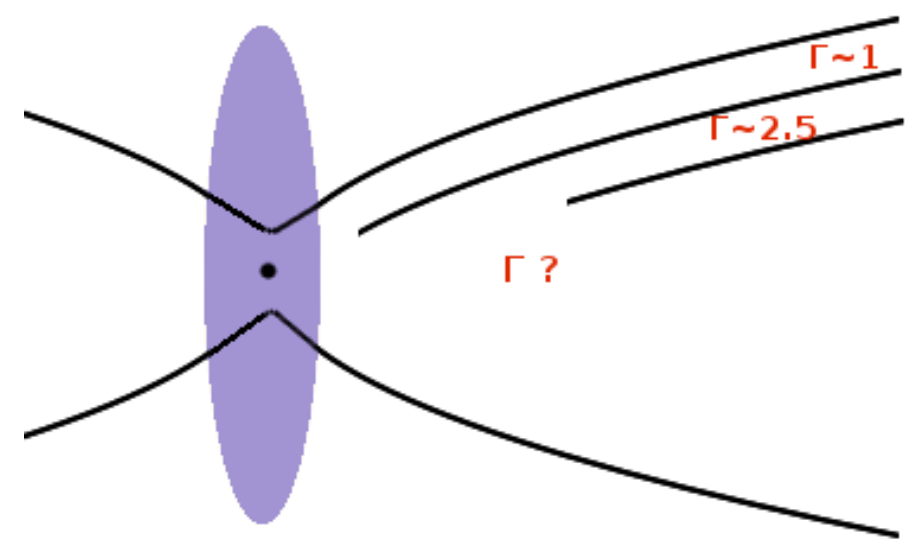

Figure 6. Simplified sketch of the stratified jet in Cygnus A. The emission is produced by a parabolically expanding, mildly relativistic disk wind with spine-sheath structure. Close to the jet axis, an even faster flow of unknown speed may exist, as suggested by the observed limb-brightening. 
Acknowledgments: We thank Shoko Koyama for carefully reading the manuscript. This research has made use of data obtained with the Global Millimeter VLBI Array (GMVA), which consists of telescopes operated by the MPIfR, IRAM, Onsala, Metsahovi, Yebes and the VLBA. The data were correlated at the correlator of the MPIfR in Bonn, Germany. The VLBA is an instrument of the National Radio Astronomy Observatory, a facility of the National Science Foundation operated under cooperative agreement by Associated Universities, Inc. The European VLBI Network is a joint facility of European, Chinese, South African, and other radio astronomy institutes funded by their national research councils.

Author Contributions: Bia Boccardi performed the analysis and wrote the manuscript. Thomas P. Krichbaum and Uwe Bach contributed to the calibration of the data and to the scientific discussion.

Conflicts of Interest: The authors declare no conflict of interest.

\section{References}

1. Vlahakis, N.; Königl, A. Magnetic Driving of Relativistic Outflows in Active Galactic Nuclei. I. Interpretation of Parsec-Scale Accelerations. Astrophys. J. 2004, 605, 656-661.

2. Lyubarsky, Y. Asymptotic Structure of Poynting-Dominated Jets. Astrophys. J. 2009, 698, 1570-1589.

3. McKinney, J.C. General relativistic magnetohydrodynamic simulations of the jet formation and large-scale propagation from black hole accretion systems. Mon. Not. R. Astron. Soc. 2006, 368, 1561-1582.

4. Komissarov, S.S.; Barkov, M.V.; Vlahakis, N.; Königl, A. Magnetic acceleration of relativistic active galactic nucleus jets. Mon. Not. R. Astron. Soc. 2007, 380, 51-70.

5. Marscher, A.P.; Jorstad, S.G.; D'Arcangelo, F.D.; Smith, P.S.; Williams, G.G.; Larionov, V.M.; Oh, H.; Olmstead, A.R.; Aller, M.F.; Aller, H.D.; et al. The inner jet of an active galactic nucleus as revealed by a radio-to- $\gamma$-ray outburst. Nature 2008, 452, 966-969.

6. Homan, D.C.; Kovalev, Y.Y.; Lister, M.L.; Ros, E.; Kellermann, K.I.; Cohen, M.H.; Vermeulen, R.C.; Zensus, J.A.; Kadler, M. Intrinsic Brightness Temperatures of AGN Jets. Astrophys. J. Lett. 2006, 642, L115-L118.

7. Sikora, M.; Begelman, M.C.; Madejski, G.M.; Lasota, J.P. Are Quasar Jets Dominated by Poynting Flux? Astrophys. J. 2005, 625, 72-77.

8. Marscher, A.P. Astrophysics: Broad escape from the abyss. Nature 2011, 477, 164-165.

9. Hada, K.; Doi, A.; Kino, M.; Nagai, H.; Hagiwara, Y.; Kawaguchi, N. An origin of the radio jet in M87 at the location of the central black hole. Nature 2011, 477, 185-187.

10. Baczko, A.K.; Schulz, R.; Kadler, M.; Ros, E.; Perucho, M.; Krichbaum, T.P.; Böck, M.; Bremer, M.; Grossberger, C.; Lindqvist, M.; et al. A Highly Magnetized Twin-Jet Base Pinpoints a Supermassive Black Hole. Astron. Astrophys. 2016, 593, A47.

11. Blandford, R.D.; Payne, D.G. Hydromagnetic flows from accretion discs and the production of radio jets. Mon. Not. R. Astron. Soc. 1982, 199, 883-903.

12. Blandford, R.D.; Znajek, R.L. Electromagnetic extraction of energy from Kerr black holes. Mon. Not. R. Astron. Soc. 1977, 179, 433-456.

13. Doeleman, S.S.; Fish, V.L.; Schenck, D.E.; Beaudoin, C.; Blundell, R.; Bower, G.C.; Broderick, A.E.; Chamberlin, R.; Freund, R.; Friberg, P.; et al. Jet-Launching Structure Resolved Near the Supermassive Black Hole in M87. Science 2012, 338, 355-358.

14. Boccardi, B.; Krichbaum, T.P.; Bach, U.; Mertens, F.; Ros, E.; Alef, W.; Zensus, J.A. The stratified two-sided jet of Cygnus A. Acceleration and collimation. Astron. Astrophys. 2016, 585, A33.

15. Boccardi, B.; Krichbaum, T.P.; Bach, U.; Bremer, M.; Zensus, J.A. First 3 mm-VLBI imaging of the two-sided jet in Cygnus A. Zooming into the launching region. Astron. Astrophys. 2016, 588, L9.

16. Tadhunter, C.; Marconi, A.; Axon, D.; Wills, K.; Robinson, T.G.; Jackson, N. Spectroscopy of the near-nuclear regions of Cygnus A: Estimating the mass of the supermassive black hole. Mon. Not. R. Astron. Soc. 2003, 342, 861-875.

17. Bach, U.; Krichbaum, T.P.; Middelberg, E.; Alef, W.; Zensus, A.J. Resolving the jet in Cygnus A. In Proceedings of The 9th European VLBI Network Symposium on The role of VLBI in the Golden Age for Radio Astronomy and EVN Users Meeting, Bologna, Italy, 23-26 September 2008; p. 108.

18. Spruit, H.C.; Foglizzo, T.; Stehle, R. Collimation of magnetically driven jets from accretion discs. Mon. Not. R. Astron. Soc. 1997, 288, 333-342. 
19. Asada, K.; Nakamura, M. The Structure of the M87 Jet: A Transition from Parabolic to Conical Streamlines. Astrophys. J. Lett. 2012, 745, L28.

20. Carilli, C.L.; Bartel, N.; Linfield, R.P. VLBI observations of the nuclear jet in Cygnus A. Astron. J. 1991, 102, 1691-1695.

21. Lobanov, A.P. Resolution limits in astronomical images. arXiv 2005, arXiv:astro-ph/0503225.

22. Ghisellini, G.; Tavecchio, F.; Maraschi, L.; Celotti, A.; Sbarrato, T. The power of relativistic jets is larger than the luminosity of their accretion disks. Nature 2014, 515, 376-378.

(C) 2017 by the authors. Licensee MDPI, Basel, Switzerland. This article is an open access article distributed under the terms and conditions of the Creative Commons Attribution (CC BY) license (http:/ / creativecommons.org/licenses/by/4.0/). 